\title{
A Regularity Criterion for the Magneto-Micropolar Fluid Equations in $\dot{B}_{\infty, \infty}^{-1}$
}

\author{
Zhihao Tang, ${ }^{1}$ Gang Wang, ${ }^{2}$ and Haiwa Guan ${ }^{3}$ \\ ${ }^{1}$ Department of Fundamentals, Henan Polytechnic Institute, Nanyang, Henan 473009, China \\ ${ }^{2}$ Shandong Transport Vocational College, Weifang, Shandong 261206, China \\ ${ }^{3}$ Department of Public Teaching, Wenzhou Vocational College of Science and Technology, Wenzhou, Zhejiang 325000, China
}

Correspondence should be addressed to Haiwa Guan; haiwaguan1234@126.com

Received 16 January 2013; Accepted 2 March 2013

Academic Editor: Fuyi Xu

Copyright (C) 2013 Zhihao Tang et al. This is an open access article distributed under the Creative Commons Attribution License, which permits unrestricted use, distribution, and reproduction in any medium, provided the original work is properly cited.

The paper is dedicated to study of the Cauchy problem for the magneto-micropolar fluid equations in three-dimensional spaces. A new logarithmically improved regularity criterion for the magneto-micropolar fluid equations is established in terms of the pressure in the homogeneous Besov space $\dot{B}_{\infty, \infty}^{-1}$.

\section{Introduction}

This paper concerns with the regularity of weak solutions to the magneto-micropolar fluid equations in three dimensions as

$$
\begin{gathered}
\partial_{t} v-(\mu+\chi) \Delta v+v \cdot \nabla v-b \cdot \nabla b+\nabla\left(p+b^{2}\right) \\
-\chi \nabla \times \omega=0, \\
\partial_{t} \omega-\gamma \Delta \omega-\kappa \nabla \operatorname{div} \omega+2 \chi \omega+v \cdot \nabla \omega-\chi \nabla \times v=0, \\
\partial_{t} b-v \Delta b+v \cdot \nabla b-b \cdot \nabla v=0, \\
\operatorname{div} v=\operatorname{div} b=0, \\
v(0, x)=v_{0}(x), \quad \omega(0, x)=\omega_{0}(x), \\
b(0, x)=b_{0}(x),
\end{gathered}
$$

where $v(t, x)=\left(v_{1}(t, x), v_{2}(t, x), v_{3}(t, x)\right) \in \mathbb{R}^{3}$ denotes the velocity of the fluid at a point $x \in \mathbb{R}^{3}, t \in[0, T), \omega(t, x) \in$ $\mathbb{R}^{3}, b(t, x) \in \mathbb{R}^{3}$, and $p(t, x) \in \mathbb{R}$ denote, respectively, the microrotational velocity, the magnetic field, and the hydrostatic pressure. $\mu, \chi, \kappa, \gamma, \nu$ are positive numbers associated to properties of the material: $\mu$ is the kinematic viscosity, $\chi$ is the vortex viscosity, $\kappa$ and $\gamma$ are spin viscosities, and $1 / \nu$ is the magnetic Reynold. $u_{0}, \omega_{0}, b_{0}$ are initial data for the velocity, the angular velocity, and the magnetic field with properties $\operatorname{div} u_{0}=0$ and $\operatorname{div} b_{0}=0$. For more detailed background, we refer the readers to [1-3].

As we know, the problem of global regularity or finite time singularity for the weak solutions of the magneto-micropolar fluid equations model with large initial data still remains unsolved since (1) includes the 3D Navier-Stokes equations. It is of interest that the regularity of the weak solutions is under preassumption of certain growth conditions. There are a lot of lectures to study the regularity of weak solutions of the magneto-micropolar fluid equations (see, [46]). The purpose of this paper is to establish a new logarithmically improved regularity criterion for the micropolar fluid equations in terms of the pressure in Besov space $\dot{B}_{\infty, \infty}^{-1}$. Now we state the main results as follows.

Theorem 1. Let $\left(v_{0}(x), \omega_{0}(x), b_{0}(x)\right) \in H^{1}\left(\mathbb{R}^{3}\right)$. Let $T>0$ and $(v, \omega, b)$ be a weak solution to the system (1). If the pressure filed $P$ satisfies the following condition:

$$
\int_{0}^{T} \frac{\|P(t, \cdot)\|_{\dot{B}_{\infty, \infty}^{-1}}^{2}}{1+\ln \left(e+\|P(t, \cdot)\|_{\dot{B}_{\infty, \infty}^{-1}}\right)} d t<\infty
$$

then the weak solution $(v, \omega, b)$ is regular on $[0, T]$. 
Remark 2. Since the space $\dot{B}_{\infty, \infty}^{-1}$ is wider than $\dot{M}_{2,3}$, so our result resolves the limit case $r=1$ in [7], which greatly improves the result in [7].

Remark 3. Since the space $\dot{B}_{\infty, \infty}^{-1}$ is wider than $L^{3 / r, \infty}$, hence our result extends and improves the recent results given by [4].

\section{Preliminaries and Lemmas}

Throughout this paper, we introduce some function spaces, notations, and important inequalities.

Let $e^{t \Delta}$ denote the heat semigroup defined by

$$
e^{t \Delta} f=K_{t} * f, \quad K_{t}=(4 \pi t)^{-3 / 2} \exp \left(-\frac{|x|^{2}}{4 t}\right)
$$

for $t>0$ and $x \in \mathbb{R}^{3}$, where $*$ denotes the convolution of functions defined on $\mathbb{R}^{3}$.

We now recall the definition of the homogeneous Besov space with negative indices $\dot{B}_{\infty, \infty}^{-\alpha}$ on $\mathbb{R}^{n}$ and the homogeneous Sobolev space $\dot{H}_{q}^{\alpha}$ of exponent $\alpha>0$. It is known (p. 192 of [8]) that $f \in \mathcal{S}^{\prime}\left(\mathbb{R}^{3}\right)$ belongs to $\dot{B}_{\infty, \infty}^{-\alpha}$ if and only if $e^{t \Delta} \in L^{\infty}$ for all $t>0$ and $t^{\alpha / 2}\left\|e^{t \Delta}\right\|_{\infty} \in L^{\infty}\left(0, \infty ; L^{\infty}\right)$. The norm of $\dot{B}_{\infty, \infty}^{-\alpha}$ is defined, up to equivalence, by

$$
\|f\|_{\dot{B}_{\infty, \infty}^{-\alpha}}=\sup _{t>0}\left(t^{\alpha / 2}\left\|e^{t \Delta}\right\|_{\infty}\right)
$$

We introduce now the homogeneous Sobolev space $\dot{H}_{q}^{\alpha}\left(\mathbb{R}^{3}\right)$, which is defined by the set of functions $f \in L^{r}\left(\mathbb{R}^{3}\right), 1 / r=$ $(1 / q)-(s / 3)$ such that $(-\Delta)^{s / 2} f \in L^{q}\left(\mathbb{R}^{3}\right)$. This space is endowed with the norm

$$
\|f\|_{\dot{H}_{q}^{\alpha}}=\left\|(-\Delta)^{s / 2} f\right\|_{L^{q}}
$$

and when $q=2$, we just let $\dot{H}_{2}^{\alpha}\left(\mathbb{R}^{3}\right)=\dot{H}^{\alpha}\left(\mathbb{R}^{3}\right)$. Additionally, we have the following inclusion relations (see, e.g., [9]):

$$
\begin{aligned}
& \dot{H}^{1 / 2}\left(\mathbb{R}^{3}\right) \subset L^{3}\left(\mathbb{R}^{3}\right) \subset L^{3, \infty}\left(\mathbb{R}^{3}\right) \subset \dot{B}_{\infty, \infty}^{-1}\left(\mathbb{R}^{3}\right), \\
& \dot{H}^{1 / 2}\left(\mathbb{R}^{3}\right) \subset L^{3}\left(\mathbb{R}^{3}\right) \subset \dot{\mathscr{M}}_{2,3}\left(\mathbb{R}^{3}\right) \subset \dot{B}_{\infty, \infty}^{-1}\left(\mathbb{R}^{3}\right)
\end{aligned}
$$

with continuous injection.

Lemma 4 (see [10]). Let $1<p<q<\infty$ and $s=\alpha((q / p)-$ $1)>0$. Then there exists a constant $C$ depending only on $\alpha, p$, and $q$ such that for all $f \in \dot{H}_{p}^{\alpha}\left(\mathbb{R}^{3}\right) \cap \dot{B}_{\infty, \infty}^{-\alpha}\left(\mathbb{R}^{3}\right)$,

$$
\|f\|_{L^{q}} \leq C\left\|(-\Delta)^{s / 2} f\right\|_{L^{p}}^{p / q}\|f\|_{\dot{B}_{\infty, \infty}^{-\alpha}}^{1-(p / q)}
$$

In particular, for $s=1, p=2$, and $q=4$, we get $\alpha=1$ and

$$
\|f\|_{L^{4}} \leq C\|f\|_{\dot{H}^{1}}^{1 / 2}\|f\|_{\dot{B}_{\infty, \infty}^{-1}}^{1 / 2} .
$$

Lemma 5 (see [11]). Let $f \in W^{1, s}\left(\mathbb{R}^{3}\right)(s \geq 1)$, and $r \geq 1$, then there exists a positive constant $C$ independent of $f$ such that

$$
\|f\|_{L^{\gamma}} \leq C\|f\|_{L^{2}}^{1-\alpha}\|\nabla f\|_{L^{2}}^{\alpha}
$$

where

$$
\alpha=\frac{(1 / r)-(1 / \gamma)}{(1 / 3)-(1 / s)-(1 / r)} \text {. }
$$

\section{Proof of Theorem 1}

For given initial data $\left(v_{0}, \omega_{0}, b_{0}\right) \in H^{1}\left(\mathbb{R}^{3}\right)$, the weak solution is the same as the local strong solution $(v, \omega, b)$ in a local interval $(0, T)$ as in the discussion of Navier-Stokes equations. For the uniqueness and existence of local strong solution, we refer to [1]. Thus, it proves that Theorem 1 is reduced to establish a priori estimates uniformly in $(0, T)$ for strong solutions. With the use of the a priori estimates, the local strong solution $(v, \omega, b)$ can be continuously extended to $t=$ $T$ by a standard process to obtain global regularity of the weak solution. Therefore, we assume that the solution $(v, \omega, b)$ is sufficiently smooth on $(0, T)$.

Proof of Theorem 1. We show that Theorem 1 holds under condition (1). To prove the theorem, we need the $L^{4}$-estimate. For this purpose, taking the inner product of the first equation of (1) with $|u|^{2} u$ and integrating by parts, it can be deduced that

$$
\begin{aligned}
& \frac{1}{4} \frac{d}{d t}\|u\|_{L^{4}}^{4}+(\mu+\chi)\||\nabla u||u|\|_{L^{2}}^{2} \\
& \quad+\frac{1}{2}(\mu+\chi)\left\|\nabla|u|^{2}\right\|_{L^{2}}^{2} \\
& \leq 2 \int_{\mathbb{R}^{3}}|P||u|^{2}|\nabla u| d x+3 \chi \int_{\mathbb{R}^{3}}|w||u|^{2}|\nabla u| d x \\
& \quad-\int_{\mathbb{R}^{3}}|b|\left|\nabla\left(|u|^{2} u\right)\right||b| d x,
\end{aligned}
$$

where we used the following relations by the divergence-free condition $\operatorname{div} u=0$ :

$$
\begin{gathered}
\int_{\mathbb{R}^{3}} u \cdot \nabla u \cdot|u|^{2} u d x=\frac{1}{2} \int_{\mathbb{R}^{3}} u \cdot \nabla|u|^{4} d x=0 \\
\int_{\mathbb{R}^{3}} \Delta u \cdot|u|^{2} u d x=-\int_{\mathbb{R}^{3}}|\nabla u|^{2}|u|^{2} d x-\left.\left.\frac{1}{2} \int_{\mathbb{R}^{3}}|\nabla| u\right|^{2}\right|^{2} d x \\
\int_{\mathbb{R}^{3}} \nabla \times \omega \cdot|u|^{2} u d x \\
=-\int_{\mathbb{R}^{3}}|u|^{2} \omega \cdot \nabla \times u d x-\int_{\mathbb{R}^{3}} \omega \cdot \nabla|u|^{2} \times u d x \\
|\nabla \times u| \leq|\nabla u|, \quad|\nabla| u|| \leq|\nabla u| .
\end{gathered}
$$


Similarly, taking the inner product of the second equation of (1) with $|\omega|^{2} \omega$ and integrating by parts, it can be inferred that

$$
\begin{aligned}
& \frac{1}{4} \frac{d}{d t}\|\omega\|_{L^{4}}^{4}+\gamma\||\nabla \omega||\omega|\|_{L^{2}}^{2}+\frac{\gamma}{2}\left\|\nabla|\omega|^{2}\right\|_{L^{2}}^{2}+k\|\operatorname{div} \omega\|_{L^{2}}^{2} \\
& +2 \chi\|\omega\|_{L^{4}}^{4} \leq 3 \chi \int_{\mathbb{R}^{3}}|u||\omega|^{2}|\nabla \omega| d x .
\end{aligned}
$$

Using an argument similar to that used in deriving the estimate (11)-(13), it can be obtained for the third equation of (1) that

$$
\begin{aligned}
& \frac{1}{4} \frac{d}{d t}\|b\|_{L^{4}}^{4}+\||\nabla b||b|\|_{L^{2}}^{2}+2\|\nabla|b||b|\|_{L^{2}}^{2} \\
& \quad \leq \int_{\mathbb{R}^{3}}|b|\left|\nabla\left(|b|^{2} b\right)\right||u| d x .
\end{aligned}
$$

Adding up (11), (13), and (14), then we obtain

$$
\begin{aligned}
\frac{1}{4} \frac{d}{d t}( & \left.\|u\|_{L^{4}}^{4}+\|\omega\|_{L^{4}}^{4}+\|b\|_{L^{4}}^{4}\right)+(\mu+\chi)\||\nabla u||u|\|_{L^{2}}^{2} \\
& +\frac{1}{2}(\mu+\chi)\left\|\nabla|u|^{2}\right\|_{L^{2}}^{2}+\gamma\||\nabla \omega||\omega|\|_{L^{2}}^{2}+\frac{\gamma}{2}\left\|\nabla|\omega|^{2}\right\|_{L^{2}}^{2} \\
& +k\|\operatorname{div} \omega\|_{L^{2}}^{2}+2 \chi\|\omega\|_{L^{4}}^{4}+\||\nabla b||b|\|_{L^{2}}^{2}+2\|\nabla|b||b|\|_{L^{2}}^{2} \\
\leq & 2 \int_{\mathbb{R}^{3}}|P||u|^{2}|\nabla u| d x+3 \chi \int_{\mathbb{R}^{3}}|w||u|^{2}|\nabla u| d x \\
& +3 \chi \int_{\mathbb{R}^{3}}|u||\omega|^{2}|\nabla \omega| d x-\int_{\mathbb{R}^{3}}|b|\left|\nabla\left(|u|^{2} u\right)\right||b| d x \\
& +\int_{\mathbb{R}^{3}}|b|\left|\nabla\left(|b|^{2} b\right)\right||u| d x \\
\triangleq & I_{1}+I_{2}+I_{3}+I_{4}+I_{5} .
\end{aligned}
$$

Applying the Hölder inequality and the Young inequality for $I_{2}$, it follows that

$$
I_{2} \leq \frac{\chi+\mu}{2}\||\nabla u||u|\|_{L^{2}}^{2}+C\left(\|u\|_{L^{4}}^{4}+\|\omega\|_{L^{4}}^{4}\right) .
$$

Arguing similarly to above, it can be derived for $I_{3}$ that

$$
I_{3} \leq \frac{\gamma}{2}\||\nabla \omega||\omega|\|_{L^{2}}^{2}+C\left(\|u\|_{L^{4}}^{4}+\|\omega\|_{L^{4}}^{4}\right) .
$$

Considering the term $I_{1}$, by virtue of the Cauchy inequality, we have

$$
I_{1} \leq\left.\left.\frac{1}{2} \int_{\mathbb{R}^{3}}|\nabla| v\right|^{2}\right|^{2} d x+\frac{1}{2} \int_{\mathbb{R}^{3}}|P|^{2}|v|^{2} d x .
$$

Let us bound the integral (1/2) $\int_{\mathbb{R}^{3}}|P|^{2}|v|^{2} d x$. Applying the divergence operator div to the first equation of (1), one formally has $P=\sum_{i, j=1}^{3} R_{i} R_{j}\left(u_{i} u_{j}-b_{i} b_{j}\right)$, where $R_{j}$ denotes the $j$ th Riesz operator. By the Calderon-Zygmund inequality, we have

$$
\|\nabla P\|_{L^{2}} \leq C\left(\||v||\nabla v|\|_{L^{2}}+\||b||\nabla b|\|_{L^{2}}\right) .
$$

With the help of (8) and (19), by the Hölder inequality and the Young inequality, we deduce that

$$
\begin{aligned}
& \frac{1}{2} \int_{\mathbb{R}^{3}}|P|^{2}|v|^{2} d x \\
& \quad \leq \frac{1}{2}\|P\|_{L^{4}}^{2}\|v\|_{L^{4}}^{2} \leq C\|\nabla P\|_{L^{2}}\|P\|_{\dot{B}_{\infty, \infty}^{-1}}\|v\|_{L^{4}}^{2} \\
& \quad \leq C\left(\||v||\nabla v|\|_{L^{2}}+\||b||\nabla b|\|_{L^{2}}\right)\|P\|_{\dot{B}_{\infty, \infty}^{-1}}\|v\|_{L^{4}}^{2} \\
& \quad=\left(\||v||\nabla v|\|_{L^{2}}+\||b||\nabla b|\|_{L^{2}}\right)\left(C\|P\|_{\dot{B}_{\infty, \infty}^{-1}}^{2}\|v\|_{L^{4}}^{4}\right)^{1 / 2} \\
& \quad \leq \frac{1}{4}\left(\||v||\nabla v|\|_{L^{2}}^{2}+\||b| \mid \nabla b\|_{L^{2}}^{2}\right)+C\|P\|_{\dot{B}_{\infty, \infty}^{-1}}^{2}\|v\|_{L^{4}}^{4} .
\end{aligned}
$$

So the term $I_{1}$ can be estimated as

$$
\begin{aligned}
I_{1} \leq & \left.\left.\frac{1}{2} \int_{\mathbb{R}^{3}}|\nabla| v\right|^{2}\right|^{2} d x+\frac{1}{4}\left(\||v||\nabla v|\|_{L^{2}}^{2}+\||b||\nabla b|\|_{L^{2}}^{2}\right) \\
& +C\|P\|_{\dot{B}_{\infty, \infty}^{-1}}^{2}\|v\|_{L^{4}}^{4} .
\end{aligned}
$$

Next we have the following estimate for the term $I_{4}$ :

$$
I_{4} \leq\left.\int_{\mathbb{R}^{3}}|b|^{2}|u||\nabla| u\right|^{2} \mid d x
$$

Since $u \in L^{2}\left(\mathbb{R}^{3}\right) \cap L^{6}\left(\mathbb{R}^{3}\right)$ and using Cauchy inequality, generalized Hölder inequality, Gagliardo-Nirenberg inequality, and Sobolev imbedding theorem, we obtain

$$
\begin{aligned}
I_{4} & \leq C\left\||b|^{2}|u|\right\|_{L^{2}}\left\|\nabla|u|^{2}\right\|_{L^{2}} \leq C\left\||b|^{2}|u|\right\|_{L^{2}}^{2}+\frac{\chi+\mu}{4}\left\|\nabla|u|^{2}\right\|_{L^{2}}^{2} \\
& \leq C\left\||b|^{2}\right\|_{L^{6}}^{2}\|u\|_{L^{3}}^{2}+\frac{\chi+\mu}{4}\left\|\nabla|u|^{2}\right\|_{L^{2}}^{2} \\
& \leq C\left\|\nabla|b|^{2}\right\|_{L^{2}}^{2}\|u\|_{L^{2}}\|\nabla u\|_{L^{2}}+\frac{\chi+\mu}{4}\left\|\nabla|u|^{2}\right\|_{L^{2}}^{2} \\
& \leq C\||b| \nabla|b|\|_{L^{2}}^{2}+\frac{\chi+\mu}{4}\left\|\nabla|u|^{2}\right\|_{L^{2}}^{2}
\end{aligned}
$$

The last term of (15) can be treated in the same way as

$$
\begin{aligned}
I_{5} & \leq\left. C \int_{\mathbb{R}^{3}}|b|^{2}|u||\nabla| b\right|^{2}\left|d x \leq C\left\||b|^{2}|u|\right\|_{L^{2}}^{2}+\frac{1}{8}\left\|\nabla|b|^{2}\right\|_{L^{2}}\right. \\
& \leq C\||b| \nabla|b|\|_{L^{2}}^{2} .
\end{aligned}
$$


Inserting the estimates (15) and (21) into (14), it follows that

$$
\begin{aligned}
\frac{d}{d t}( & \left.\|v\|_{4}^{4}+\|\omega\|_{4}^{4}+\|b\|_{4}^{4}\right) \\
\leq & C\|P\|_{\dot{B}_{\infty, \infty}^{-1}}^{2}\|v\|_{L^{4}}^{4}+C\left(\|v\|_{4}^{4}+\|\omega\|_{4}^{4}+\|b\|_{4}^{4}\right) \\
\leq & C\|P\|_{\dot{B}_{\infty, \infty}^{-1}}^{2}\left(\|v\|_{4}^{4}+\|\omega\|_{4}^{4}\right)+C\left(\|v\|_{4}^{4}+\|\omega\|_{4}^{4}+\|b\|_{4}^{4}\right) \\
\leq & C\left(1+\frac{\|P(t, \cdot)\|_{\dot{B}_{\infty, \infty}^{-1}}^{2}}{1+\ln \left(e+\|P(t, \cdot)\|_{\dot{B}_{\infty, \infty}^{-1}}\right)}\right) \\
& \times\left[1+\ln \left(e+\|P(t, \cdot)\|_{\dot{B}_{\infty, \infty}^{-1}}^{-1}\right)\right]\left(\|v\|_{4}^{4}+\|\omega\|_{4}^{4}+\|b\|_{4}^{4}\right) \\
\leq & C\left(1+\frac{\|P(t, \cdot)\|_{\dot{B}_{\infty, \infty}^{-1}}^{2}}{1+\ln \left(e+\|P(t, \cdot)\|_{\dot{B}_{\infty, \infty}^{-1}}\right)}\right) \\
& \times\left[1+\ln \left(e+\|P(t, \cdot)\|_{L^{3}}\right)\right]\left(\|v\|_{4}^{4}+\|\omega\|_{4}^{4}+\|b\|_{4}^{4}\right) \\
\leq & C\left(1+\frac{\|P(t, \cdot)\|_{\dot{B}_{\infty, \infty}^{-1}}^{2}}{1+\ln \left(e+\|P(t, \cdot)\|_{\dot{B}_{\infty, \infty}^{-1}}\right)}\right) \\
\leq & C\left(1+\frac{\|P(t, \cdot)\|_{\dot{B}_{\infty, \infty}^{-1}}^{2}}{1+\ln \left(e+\|P(t, \cdot)\|_{\dot{B}_{\infty, \infty}^{-1}}\right)}\right) \\
& \times\left[1+\ln \left(e+\|v(t, \cdot)\|_{L^{6}}^{2}\right)\right]\left(\|v\|_{4}^{4}+\|\omega\|_{4}^{4}+\|b\|_{4}^{4}\right) \\
& \times[1+\ln (e+y(t))]\left(\|v\|_{4}^{4}+\|\omega\|_{4}^{4}+\|b\|_{4}^{4}\right),
\end{aligned}
$$

where $y(t)$ is defined by

$$
y(t)=: \sup _{T_{0} \leq s \leq t}\left(\left\|\Lambda^{3} v\right\|_{L^{2}}^{2}+\left\|\Lambda^{3} \omega\right\|_{L^{2}}^{2}+\left\|\Lambda^{3} b\right\|_{L^{2}}^{2}\right) .
$$

Applying Gronwall's inequality on (25) for the interval $\left[T_{0}, t\right]$, one has

$$
\begin{aligned}
\sup _{T_{0} \leq s \leq t}\left(\|v\|_{4}^{4}+\|\omega\|_{4}^{4}+\|b\|_{4}^{4}\right) & \leq C_{0} \exp (C \varepsilon(1+\ln (e+y(t)))) \\
& \leq C_{0} \exp (2 C \varepsilon \ln (e+y(t))) \\
& \leq C_{0}(e+y(t))^{2 C \varepsilon}
\end{aligned}
$$

provided that

$$
\int_{T_{0}}^{t} \frac{\|P(t, \cdot)\|_{\dot{B}_{\infty, \infty}^{-1}}^{2}}{1+\ln \left(e+\|P(t, \cdot)\|_{\dot{B}_{\infty, \infty}^{-1}}\right)} d s<\varepsilon \ll 1,
$$

where $C_{0}$ is a positive constant depending on $T_{0}$.

Next we will estimate the $L^{2}$-norm of $\nabla v, \nabla \omega$, and $\nabla b$. We multiply both sides of the first equation of (1) by $(-\Delta v)$, the second equation of (1) by $(-\Delta \omega)$, and the third equation of (1) by $(-\Delta b)$, by integration by parts over $\mathbb{R}^{3}$, we get

$$
\begin{aligned}
\frac{1}{2} \frac{d}{d t} & \|\nabla v\|_{L^{2}}^{2}+\|\Delta v\|_{L^{2}}^{2} \\
= & \int_{\mathbb{R}^{3}}(v \cdot \nabla) v \cdot \Delta v d x \\
& +\int_{\mathbb{R}^{3}}(b \cdot \nabla) b \cdot \Delta v d x-\int_{\mathbb{R}^{3}} \operatorname{curl} \omega \Delta v d x \\
\leq & \|v\|_{L^{4}}\|\nabla v\|_{L^{4}}\|\Delta v\|_{L^{2}} \\
& +\|b\|_{L^{4}}\|\nabla b\|_{L^{4}}\|\Delta v\|_{L^{2}}+\|\nabla \omega\|_{L^{2}}\|\Delta v\|_{L^{2}} \\
\leq & \|v\|_{L^{4}}\|v\|_{L^{2}}^{1 / 8}\|\Delta v\|_{L^{2}}^{7 / 8}\|\Delta v\|_{L^{2}} \\
& +\|b\|_{L^{4}}\|b\|_{L^{2}}^{1 / 8}\|\Delta b\|_{L^{2}}^{7 / 8}\|\Delta v\|_{L^{2}} \\
& +\frac{1}{16}\|\Delta v\|_{L^{2}}^{2}+C\|\nabla \omega\|_{L^{2}}^{2} \\
\leq & \frac{1}{8}\|\Delta v\|_{L^{2}}^{2}+\frac{1}{8}\|\Delta \omega\|_{L^{2}}^{2}+\frac{1}{8}\|\Delta b\|_{L^{2}}^{2} \\
& +C\|b\|_{L^{2}}^{16}\|b\|_{L^{2}}^{2}+C\|v\|_{L^{4}}^{16}\|v\|_{L^{2}}^{2}+C\|\omega\|_{L^{2}}^{2}, \\
\frac{1}{2} \frac{d}{d t} & \|\nabla \omega\|_{L^{2}}^{2}+\|\Delta \omega\|_{L^{2}}^{2}+\|\nabla \operatorname{div} \omega\|_{L^{2}}^{2}+2\|\nabla \omega\|_{L^{2}}^{2} \\
= & \int(v \cdot \nabla) \omega \cdot \Delta \omega d x-\int \operatorname{curl} v \Delta \omega d x \\
\leq & \|v\|_{L^{4}}\|\nabla \omega\|_{L^{4}}\|\Delta \omega\|_{L^{2}}+\|\nabla v\|_{L^{2}}\|\Delta \omega\|_{L^{2}} \\
\leq & \|v\|_{L^{4}}\|\omega\|_{L^{2}}^{1 / 8}\|\Delta \omega\|_{L^{2}}^{7 / 8}\|\Delta \omega\|_{L^{2}} \\
& +C\|v\|_{L^{2}}^{1 / 2}\|\Delta v\|_{L^{2}}^{1 / 2}\|\Delta \omega\|_{L^{2}} \\
&
\end{aligned}
$$

$$
\begin{aligned}
\frac{1}{2} & \frac{d}{d t}\|\nabla b\|_{L^{2}}^{2}+\|\Delta b\|_{L^{2}}^{2} \\
= & \int_{\mathbb{R}^{3}}(v \cdot \nabla b) \Delta b d x-\int_{\mathbb{R}^{3}}(b \cdot \nabla v) \Delta b d x \\
\leq & \|v\|_{L^{4}}\|\nabla b\|_{L^{4}}\|\Delta b\|_{L^{2}}+\|b\|_{L^{4}}\|\nabla v\|_{L^{4}}\|\Delta b\|_{L^{2}} \\
\leq & \|v\|_{L^{4}}\|b\|_{L^{2}}^{1 / 8}\|\Delta b\|_{L^{2}}^{7 / 8}\|\Delta v\|_{L^{2}} \\
& +\|b\|_{L^{4}}\|v\|_{L^{2}}^{1 / 8}\|\Delta v\|_{L^{2}}^{7 / 8}\|\Delta b\|_{L^{2}} \\
\leq & \frac{1}{8}\|\Delta v\|_{L^{2}}^{2}+\frac{1}{8}\|\Delta b\|_{L^{2}}^{2}+C\|v\|_{L^{4}}^{16}\|b\|_{L^{2}}^{2}+C\|b\|_{L^{4}}^{16}\|v\|_{L^{2}}^{2},
\end{aligned}
$$

where we have used the Gagliardo-Nirenberg inequality:

$$
\|\nabla f\|_{L^{4}} \leq C\|f\|_{L^{2}}^{1 / 8}\|\Delta f\|_{L^{2}}^{7 / 8} .
$$


Combining (29), (30), and (31) and using the definition of the weak solution, we deduce that

$$
\begin{aligned}
\|\nabla v\|_{L^{2}}^{2}+\|\nabla \omega\|_{L^{2}}^{2}+\|\nabla b\|_{L^{2}}^{2} & \\
\leq & C C_{0}(e+y(t))^{6 C \varepsilon}\left(t-T_{0}\right) \\
& +\left\|\nabla v\left(\cdot, T_{0}\right)\right\|_{L^{2}}^{2}+\left\|\nabla \omega\left(\cdot, T_{0}\right)\right\|_{L^{2}}^{2} .
\end{aligned}
$$

Finally we go to the estimate for $H^{3}$-norm of $v, \omega$, and $b$. In the following calculations, we will use the following commutator estimate due to Kato and Ponce [12]:

$$
\begin{aligned}
& \left\|\Lambda^{s}(f g)-f \Lambda^{s} g\right\|_{L^{p}} \\
& \quad \leq\left(\|\nabla f\|_{L^{p_{1}}}\left\|\Lambda^{s-1} g\right\|_{L^{q_{1}}}+\left\|\Lambda^{s} f\right\|_{L^{p_{2}}}\|g\|_{L^{q_{2}}}\right),
\end{aligned}
$$

with $s>0, \Lambda^{s}=(-\Delta)^{s / 2}$ and $(1 / p)=\left(1 / p_{1}\right)+\left(1 / q_{1}\right)=$ $\left(1 / p_{2}\right)+\left(1 / q_{2}\right)$. Taking the operation $\Lambda^{3}$ on both sides of $(1)$, then multiplying them by $\Lambda^{3} v, \Lambda^{3} \omega$, and $\Lambda^{3} b$, and integrating by parts over $\mathbb{R}^{3}$, we have

$$
\begin{aligned}
& \frac{1}{2} \frac{d}{d t} \int_{\mathbb{R}^{3}}\left|\Lambda^{3} v\right|^{2}+\left|\Lambda^{3} \omega\right|^{2}+\left|\Lambda^{3} b\right|^{2} d x \\
& \quad+\int_{\mathbb{R}^{3}}\left|\Lambda^{4} v\right|^{2} d x+\int_{\mathbb{R}^{3}}\left|\Lambda^{4} \omega\right|^{2} d x+\int_{\mathbb{R}^{3}}\left|\Lambda^{4} b\right|^{2} d x \\
& \quad+\int_{\mathbb{R}^{3}}\left|\Lambda^{3} \operatorname{div} v\right|^{2} d x+2 \int_{\mathbb{R}^{3}}\left|\Lambda^{3} \omega\right|^{2} d x \\
& =-\int_{\mathbb{R}^{3}}\left[\Lambda^{3}(v \cdot \nabla v)-v \cdot \nabla \Lambda^{3} v\right] \Lambda^{3} v d x \\
& \quad+\int_{\mathbb{R}^{3}} \Lambda^{3} \operatorname{curl} \omega \cdot \Lambda^{3} v d x \\
& \quad-\int_{\mathbb{R}^{3}}\left[\Lambda^{3}(v \cdot \nabla \omega)-v \cdot \nabla \Lambda^{3} \omega\right] \Lambda^{3} \omega d x \\
& \quad+\int_{\mathbb{R}^{3}} \Lambda^{3} \operatorname{curl} v \cdot \Lambda^{3} \omega d x \\
& \quad+\int_{\mathbb{R}^{3}}\left[\Lambda^{3}(b \cdot \nabla b)-b \cdot \nabla \Lambda^{3} b\right] \Lambda^{3} v d x \\
& \quad-\int_{\mathbb{R}^{3}}\left[\Lambda^{3}(v \cdot \nabla b)-v \cdot \nabla \Lambda^{3} b\right] \Lambda^{3} b d x \\
& \quad+\int_{\mathbb{R}^{3}}\left[\Lambda^{3}(b \cdot \nabla v)-b \cdot \nabla \Lambda^{3} v\right] \Lambda^{3} b d x \\
& \equiv A_{1}+A_{2}+A 3+A_{4}+A_{5}+A_{6}+A_{7}
\end{aligned}
$$

Hence $A_{1}$ can be estimated as

$$
\begin{aligned}
A_{1} & \leq C\|\nabla v\|_{L^{3}}\left\|\Lambda^{3} v\right\|_{L^{3}}^{2} \leq C\|\nabla v\|_{L^{2}}^{13 / 2}\left\|\Lambda^{3} v\right\|_{L^{2}}^{1 / 4}\left\|\Lambda^{4} v\right\|_{L^{2}}^{5 / 3} \\
& \leq \frac{1}{6}\left\|\Lambda^{4} v\right\|_{L^{2}}^{2}+C\|\nabla v\|_{L^{2}}^{13 / 2}\left\|\Lambda^{3} v\right\|_{L^{2}}^{3 / 2},
\end{aligned}
$$

where we used (33) with $s=3, p=3 / 2, p_{1}=q_{1}=p_{2}=$ $q_{2}=3$ and the following inequalities:

$$
\begin{gathered}
\|\nabla v\|_{L^{3}} \leq C\|\nabla v\|_{L^{2}}^{3 / 4}\left\|\Lambda^{3} v\right\|_{L^{2}}^{1 / 4}, \\
\left\|\Lambda^{3} v\right\|_{L^{3}} \leq C\|\nabla v\|_{L^{2}}^{1 / 6}\left\|\Lambda^{4} v\right\|_{L^{2}}^{5 / 6} .
\end{gathered}
$$

If we use the existing estimate (31) for $T_{0}<t<T$, (36) reduces to

$$
A_{1} \leq \frac{1}{6}\left\|\Lambda^{4} v\right\|_{L^{2}}^{2}+C C_{0}(e+y(t))^{(3 / 4)+(39 / 2) C \varepsilon} .
$$

Using (37) again, we have

$$
\begin{aligned}
A_{3}+A_{5}+A_{6}+A_{7} \leq & \frac{1}{6}\left(\left\|\Lambda^{4} v\right\|_{L^{2}}^{2}+\left\|\Lambda^{4} \omega\right\|_{L^{2}}^{2}+\left\|\Lambda^{4} b\right\|_{L^{2}}^{2}\right) \\
& +C C_{0}(e+y(t))^{(3 / 4)+(39 / 2) C \varepsilon} .
\end{aligned}
$$

For $A_{2}$ and $A_{4}$, we have

$$
\begin{aligned}
A_{2}+A_{4} & \leq \frac{1}{6}\left(\left\|\Lambda^{4} v\right\|_{L^{2}}^{2}+\left\|\Lambda^{4} \omega\right\|_{L^{2}}^{2}\right)+C\left(\left\|\Lambda^{3} v\right\|_{L^{2}}^{2}+\left\|\Lambda^{3} \omega\right\|_{L^{2}}^{2}\right) \\
& \leq \frac{1}{6}\left(\left\|\Lambda^{4} v\right\|_{L^{2}}^{2}+\left\|\Lambda^{4} \omega\right\|_{L^{2}}^{2}\right)+C C_{0}(e+y(t)) .
\end{aligned}
$$

Inserting the above estimates (38)-(40) into (35), we obtain

$$
\begin{aligned}
& \frac{d}{d t} \int_{\mathbb{R}^{3}}\left|\Lambda^{3} v\right|^{2}+\left|\Lambda^{3} \omega\right|^{2} d x \\
& \quad \leq C C_{0}(e+y(t))^{(3 / 4)+(39 / 2) C \varepsilon}+C C_{0}(e+y(t)) .
\end{aligned}
$$

Gronwall's inequality implies the boundness of $H^{3}$-norm of $v, \omega$, and $b$ provided that $39 C \varepsilon<(1 / 2)$, which can be achieved by the absolute continuous property of integral (2). This completes the proof of Theorem 1 .

\section{Acknowledgment}

The authors thank Professor Xiaohong Fan for his profitable discussion and suggestions.

\section{References}

[1] G. P. Galdi and S. Rionero, "A note on the existence and uniqueness of solutions of the micropolar fluid equations," International Journal of Engineering Science, vol. 15, no. 2, pp. 105-108, 1977.

[2] M. A. Rojas-Medar, "Magneto-micropolar fluid motion: existence and uniqueness of strong solution," Mathematische Nachrichten, vol. 188, pp. 301-319, 1997.

[3] E. E. Ortega-Torres and M. A. Rojas-Medar, "Magneto-micropolar fluid motion: global existence of strong solutions," $A b$ stract and Applied Analysis, vol. 4, no. 2, pp. 109-125, 1999.

[4] B. Yuan, "On regularity criteria for weak solutions to the micropolar fluid equations in Lorentz space," Proceedings of the American Mathematical Society, vol. 138, no. 6, pp. 2025-2036, 2010. 
[5] B. Yuan, "Regularity of weak solutions to magneto-micropolar fluid equations," Acta Mathematica Scientia. Series B, vol. 30, no. 5, pp. 1469-1480, 2010.

[6] F. Xu, "Regularity criterion of weak solution for the 3D magneto-micropolar fluid equations in Besov spaces," Communications in Nonlinear Science and Numerical Simulation, vol. 17, no. 6, pp. 2426-2433, 2012.

[7] J. Geng, X. Chen, and S. Gala, "On regularity criteria for the 3D magneto-micropolar fluid equations in the critical MorreyCampanato space," Communications on Pure and Applied Analysis, vol. 10, no. 2, pp. 583-592, 2011.

[8] H. Triebel, Interpolation Theory, Function Spaces, Differential Operators, vol. 18, North-Holland Publishing, Amsterdam, The Netherlands, 1978.

[9] Z. M. Chen and Z. Xin, "Homogeneity criterion for the NavierStokes equations in the whole spaces," Journal of Mathematical Fluid Mechanics, vol. 3, no. 2, pp. 152-182, 2001.

[10] Y. Meyer, P. Gerard, and F. Oru, "Inégalités de Sobolev précisées," in Séminaire sur les Équations aux Dérivées Partielles, p. 8, Polytechnique, Palaiseau, France, 1997.

[11] O. A. Ladyženskaja, V. A. Solonnikov, and N. N. Ural'tseva, Linear and Quasilinear Equations of Parabolic Type, Translations of Mathematical Monographs, American Mathematical Society, Providence, RI, USA, 1968.

[12] T. Kato and G. Ponce, "Commutator estimates and the Euler and Navier-Stokes equations," Communications on Pure and Applied Mathematics, vol. 41, no. 7, pp. 891-907, 1988. 


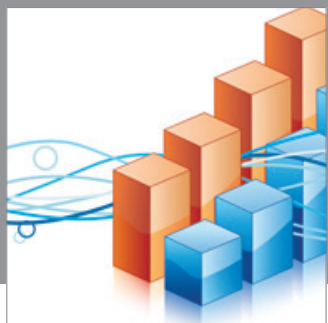

Advances in

Operations Research

mansans

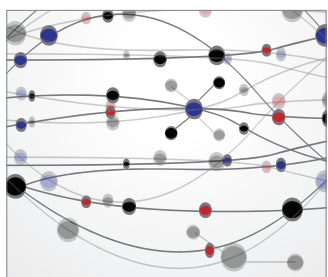

The Scientific World Journal
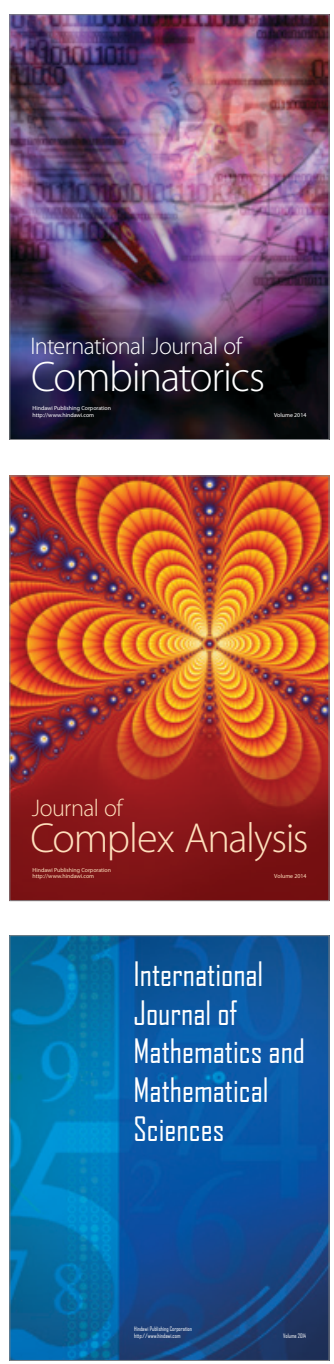
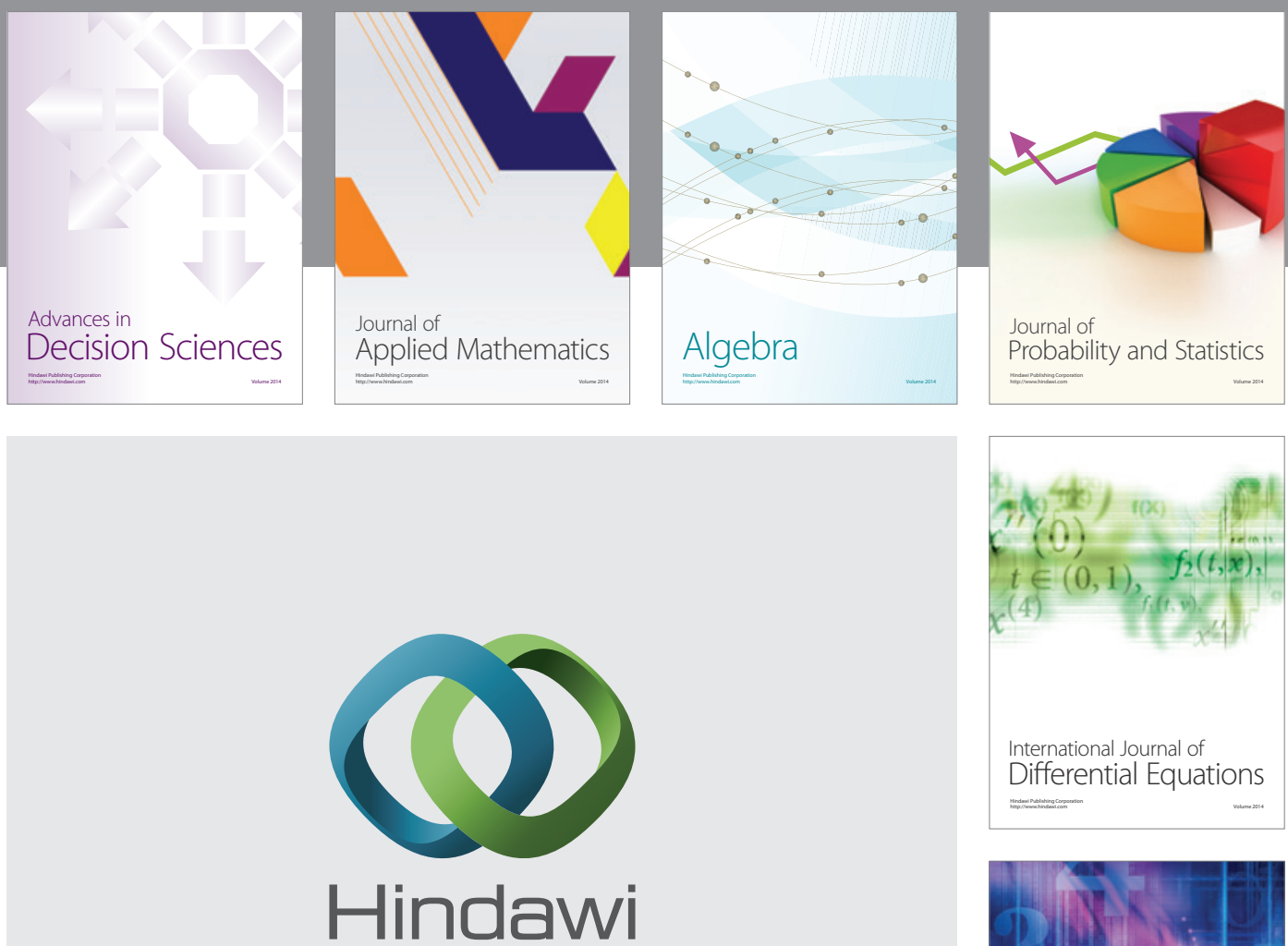

Submit your manuscripts at http://www.hindawi.com
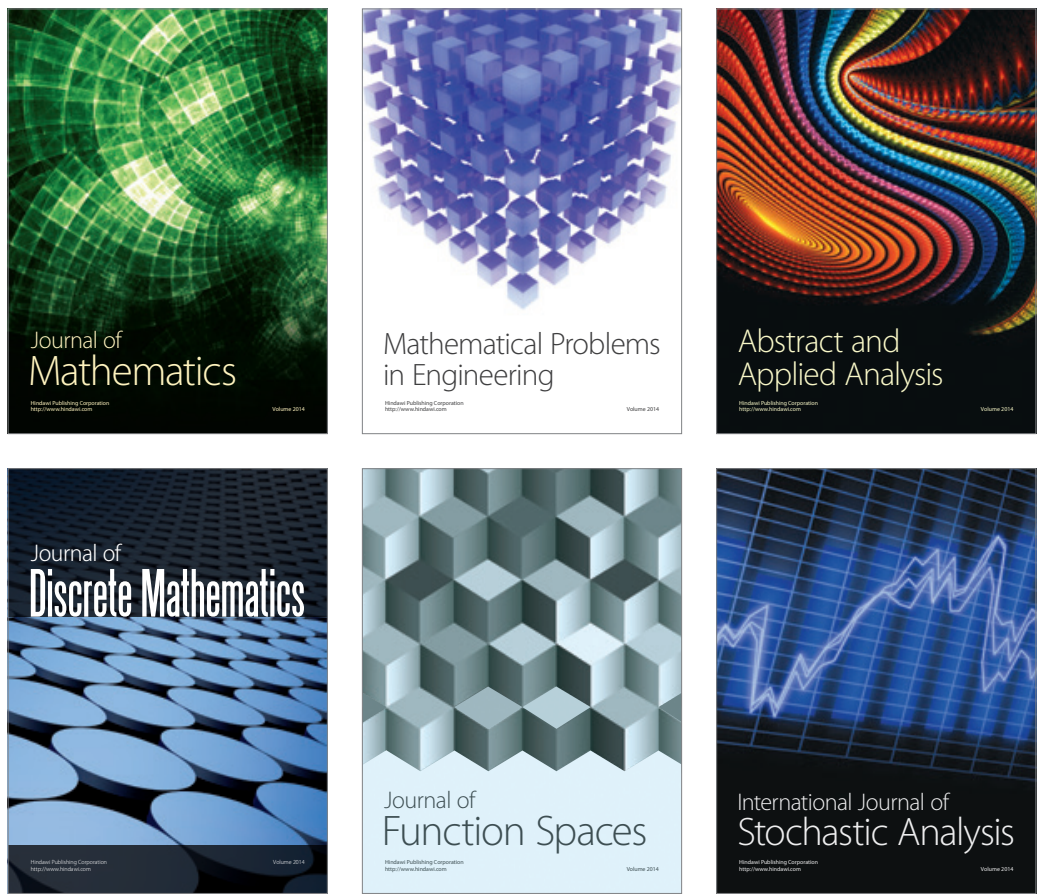

Journal of

Function Spaces

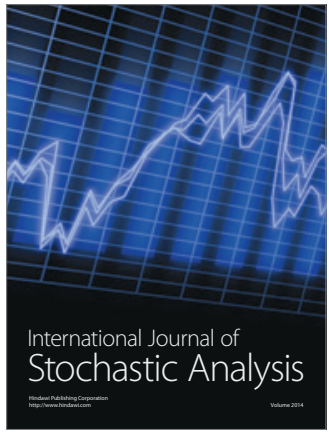

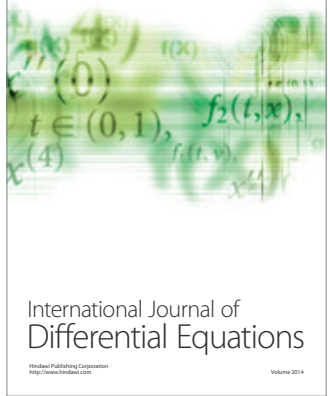
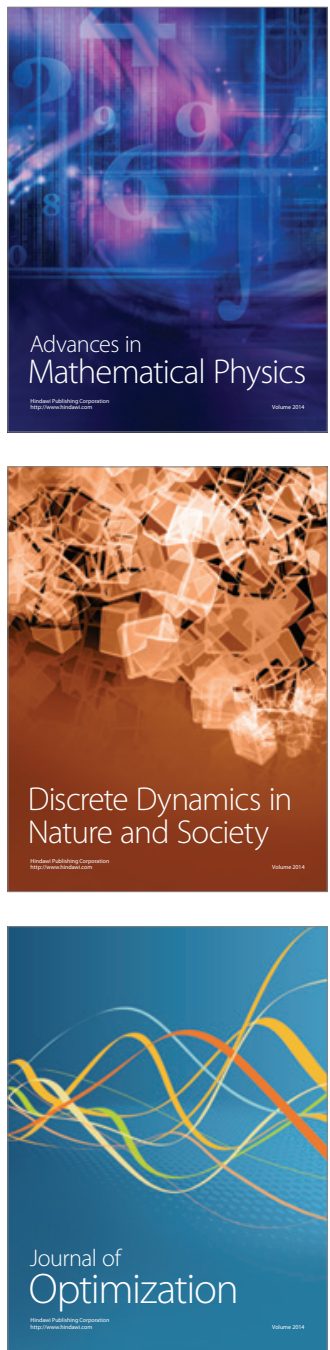